\title{
Layered Semiconductors and Related Systems
}

\author{
H. STARNBERG* \\ Department of Physics, Göteborg University \\ and Chalmers University of Technology, 41296 Göteborg, Sweden \\ The general properties of the layered transition metal dichalcogenides \\ and the possibility to modify these materials by intercalation are reviewed. \\ Examples are given of experimental results obtained by using angle-resolved \\ photoelectron spectroscopy and very-low-energy electron diffraction. The \\ possibility to use layered semiconductors as model systems in studies of e.g. \\ Schottky barriers and surface photovoltage is exemplified by the $\mathrm{Rb} / \mathrm{WSe}_{2}$ \\ system. Attention is also paid to the use of van der Waals epitaxy in inter- \\ face studies, and its possible practical applications. The potential of layered \\ semiconductors like $\mathrm{WSe}_{2}$ in solar cell applications is also mentioned.
}

PACS numbers: 71.20.Nr, 71.20.Tx, 79.60.Bm, 79.60.Jv

\section{Introduction}

The layered transition metal dichalcogenides (TMDCs) have attracted much attention in recent decades from both fundamental and applied points of view. An important reason for this interest is that their low-dimensional character is manifested through a number of unusual properties, which are theoretically challenging as well as practically useful [1].

\section{General properties of layered TMDCs}

The layered TMDCs all have the formal composition $\mathrm{TX}_{2}$, where $\mathrm{T}$ is a transition metal and $\mathrm{X}$ is $\mathrm{S}$, Se or Te. The three-dimensional crystal structure is basically a stack of layers, where each layer consists of a sheet of hexagonally arranged $\mathrm{T}$ atoms, sandwiched between two similar sheets of $\mathrm{X}$ atoms. Each $\mathrm{T}$ atom is thereby surrounded by six neighbouring $X$ atoms, and depending on the

*e-mail: starn@fy.chalmers.se 
positioning of the $\mathrm{X}$ sheets, the coordination of the $\mathrm{T}$ atoms is either octahedral $(\mathrm{OH})$ or trigonal prismatic (TP), as illustrated in Fig. 1. The internal bonds in the layers are strong, of mixed ionic/covalent character. In contrast to this, the bonding between the layers is weak, mainly of van der Waals type, and the space between two neighbouring layers is often referred to as the van der Waals gap.
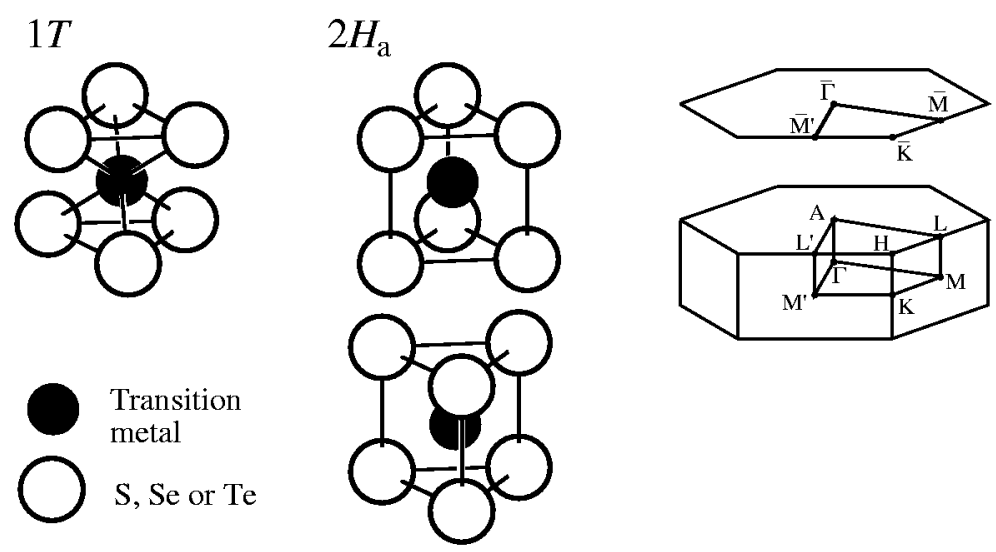

Fig. 1. The $1 T$ and $2 H_{a}$ structures together with their surface and bulk Brillouin zones. For the $1 T$ structure the "primed" and "unprimed" symmetry points are inequivalent, due to the overall trigonal symmetry of this structure.

In addition to the two different possibilities for the internal layer structure, the layers may be stacked in a number of ways. The different structures are given labels such as $1 T, 2 H$ or $6 R$, where the number specifies the perpendicular repeat distance (counted in layers) and the letter tells us whether the overall symmetry of the crystal is trigonal, hexagonal or rhombohedral. In the case of different structures ending up with the same label, one may add subscripts $(a, b, \ldots)$ to distinguish them. Two common structures are shown in Fig. 1, together with the corresponding bulk and surface Brillouin zones. Polytypism, i.e. that the same compound may appear with a number of different layered structures, is common among TMDCs. An example of this is $\mathrm{TaS}_{2}$ which is known to adopt at least five different structures: $1 T, 2 H_{a}, 3 R, 4 H_{b}$, and $6 R$.

\section{Electronic structure of TMDCs}

The electronic structure is similar for all TMDCs, and may to a first approximation be described by a generic band structure [2-4]: in the bottom a narrow chalcogen $s$ band, separated by a wide gap from a chalcogen $p$ band reaching up to or close to the Fermi energy. Next a narrow gap followed by a transition metal $d$ band, another gap, and on top of that a band composed of higher transition metal $s$ and $p$ states. The orbital labels given here refer to the states that dominate in 
each band, but in reality the characters are mixed by hybridization. In fact, the occupied chalcogen $p$ band and the unoccupied higher transition metal $s, p$ band form a bonding/antibonding combination and are thus responsible for the strong internal bonding in the layer. The transition metal $d$ band is not directly involved in the bonding, but the extent to which this band is filled is of crucial importance for the electronic properties. This simple picture is subject to modifications, however. In some cases there is a small overlap between the chalcogen $p$ bands and the transition metal $d$ band, and in materials with TP coordination a $d_{z^{2}}$ band may split off from the remaining transition metal $d$ band, resulting in an additional band gap. Figure 2 shows the schematic band structures for (a) $\mathrm{TiS}_{2}$ (with $\mathrm{OH}$ coordination) and (b) $\mathrm{MoS}_{2}$ (with TP coordination). In $\mathrm{TiS}_{2}$ the number of valence electrons per unit cell is exactly that required to fill the $\mathrm{S} 3 p$ band, while the Ti $3 d$ band remains empty. With the Fermi level situated in the small gap between these bands, $\mathrm{TiS}_{2}$ consequently is a narrow-gap semiconductor. In $\mathrm{TiSe}_{2}$ there is a small overlap instead of a gap between the Ti $3 d$ and Se $4 p$ bands, which results in semimetallic behaviour for this closely related compound. $\mathrm{VSe}_{2}$ has the same structure $(1 T)$ as $\mathrm{TiS}_{2}$ and $\mathrm{TiSe}_{2}$, but with one more electron per unit cell it becomes a narrow-band metal with a partial filling of the V $3 d$ band. In $\mathrm{MoS}_{2}$ the TP coordination creates a separate Mo $4 d_{z_{2}}$ band which is completely filled, while the remaining Mo $4 d$ band is empty. Therefore $\mathrm{MoS}_{2}$ is a semiconductor, but with a different type of band gap than $\mathrm{TiS}_{2}$.
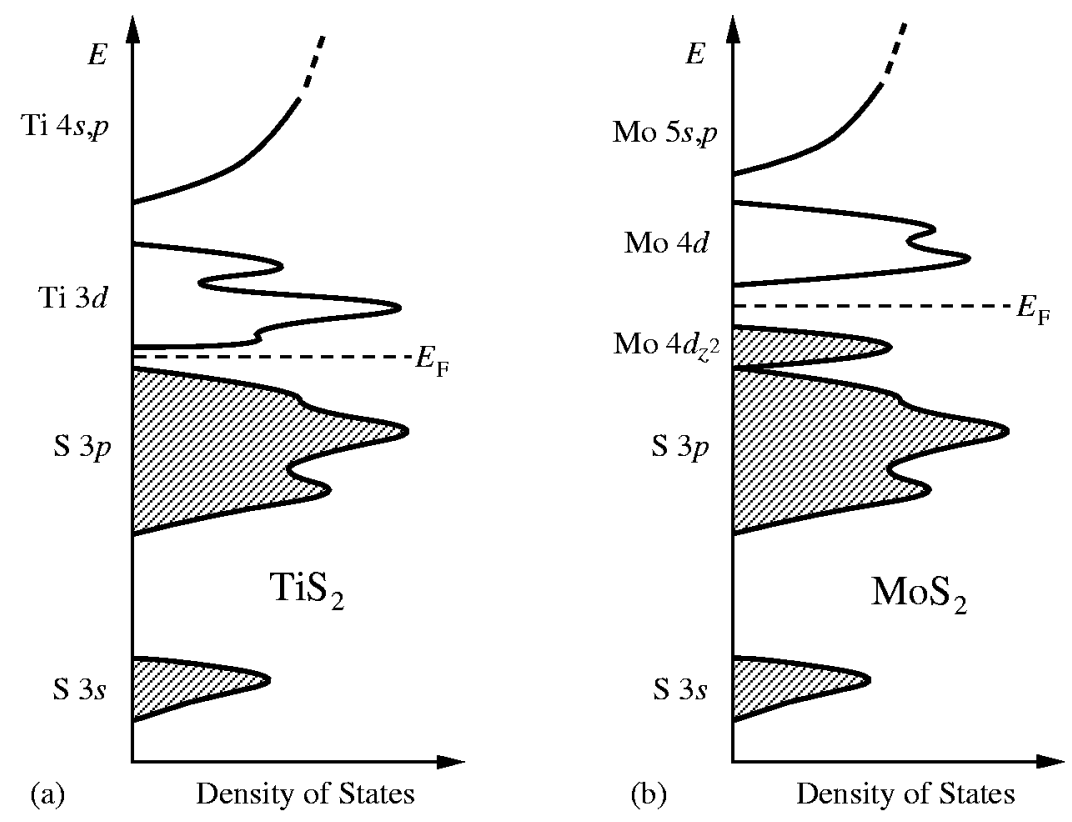

Fig. 2. Schematic electronic band structure of $\mathrm{TiS}_{2}$ and $\mathrm{MoS}_{2}$. 
Some TMDCs deviate from the sketched simple band picture by the occurrence of charge density waves with associated periodic lattice distortions [5]. This is usually attributed to the low-dimensional character of these materials, which favours Fermi surface nesting and phonon anomalies that may drive such phase transformations.

The electronic structure of TMDCs have been extensively studied by angle-resolved photoelectron spectroscopy (ARPES) [6, 7]. The principles of this technique are schematically shown in Fig. 3: As a surface is hit by incident UV or X-ray radiation, photoelectrons are emitted and their kinetic energy is measured in an energy analyser. The resulting spectrum shows the emission rate in a particular direction as a function of the energy. Provided that the photon energy is known, one easily obtains the energy of the electron prior to excitation. As the photoelectron passes through the surface potential barrier, its wave vector is changed, but $k_{\|}$, the component parallel to the surface, is conserved and can be calculated from the measured kinetic energy and the emission angle. The only quantity now missing from a complete determination of the corresponding band structure point is the

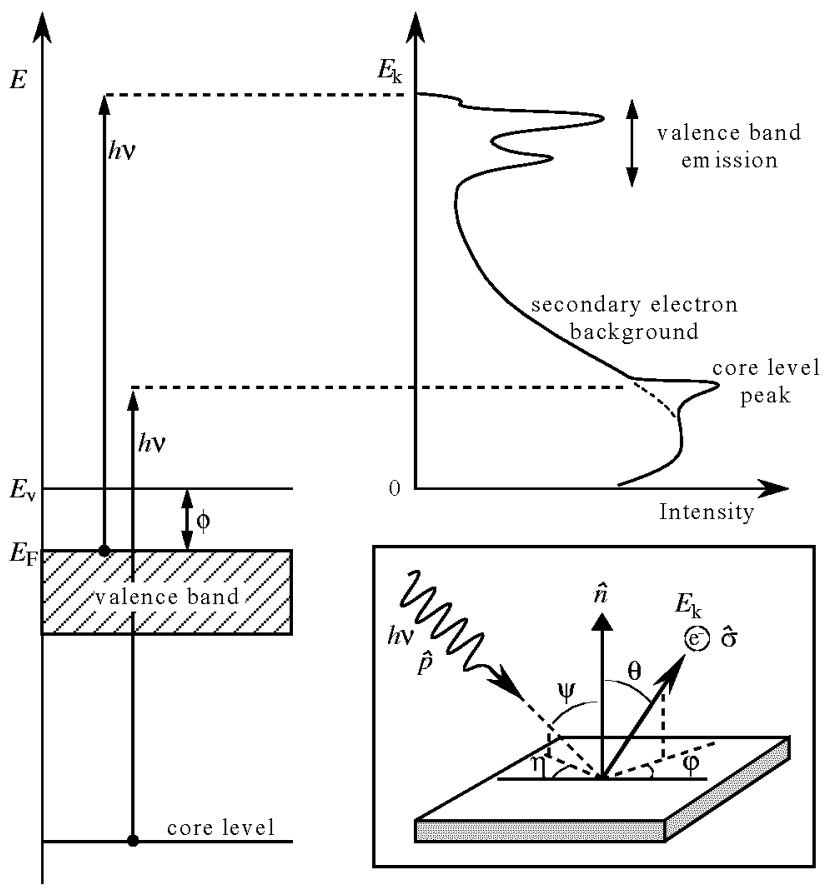

Fig. 3. Principles of ARPES: The energy level diagram (left side) illustrates photoemission from both the valence band and from a core level, which gives rise to peaks in the corresponding energy distribution curve (EDC) (right side). An EDC is normally displayed with $E_{k}$ along the horizontal axis, but here the axes have been reversed to stress the relation to the energy level diagram. The insert shows the full set of parameters in photoemission. 
surface-perpendicular wave vector component $k_{\perp}$, but there are several ways to find this, at least approximately. In TMDCs this issue is simplified by the fact that most of the bands depend only weakly on $k_{\perp}$, so that the experimental results in many cases can be compared directly with calculated bands. Thus, apart from the fundamental interest in TMDCs, there are also practical reasons for their prevalence as model systems in ARPES. Another practical advantage of TMDCs, in this context, is that high quality surfaces are easily obtained by cleavage of the crystals along the layers. Thanks to the absence of dangling bonds, these surfaces are inert and stay clean for extended time periods. As a typical example of ARPES results from TMDCs, Fig. 4 shows spectra measured from $\mathrm{ZrSe}_{2}$ at different polar angles

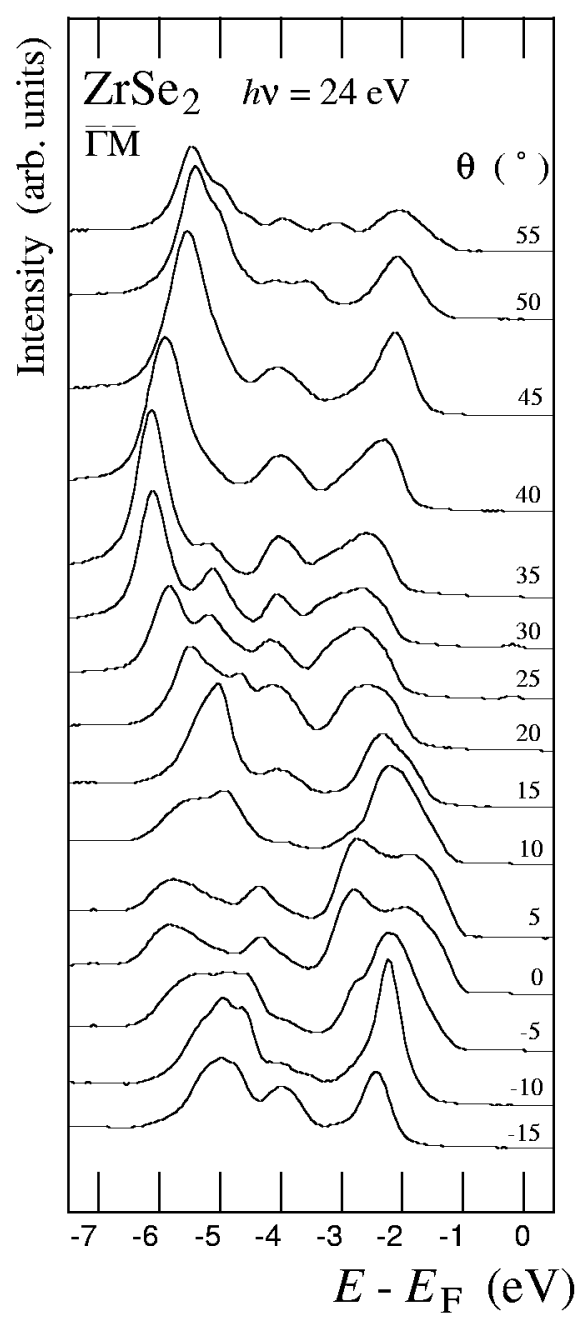

Fig. 4. Angular series of photoelectron spectra measured from $\mathrm{ZrSe}_{2}$ with $h \nu=24 \mathrm{eV}$. 


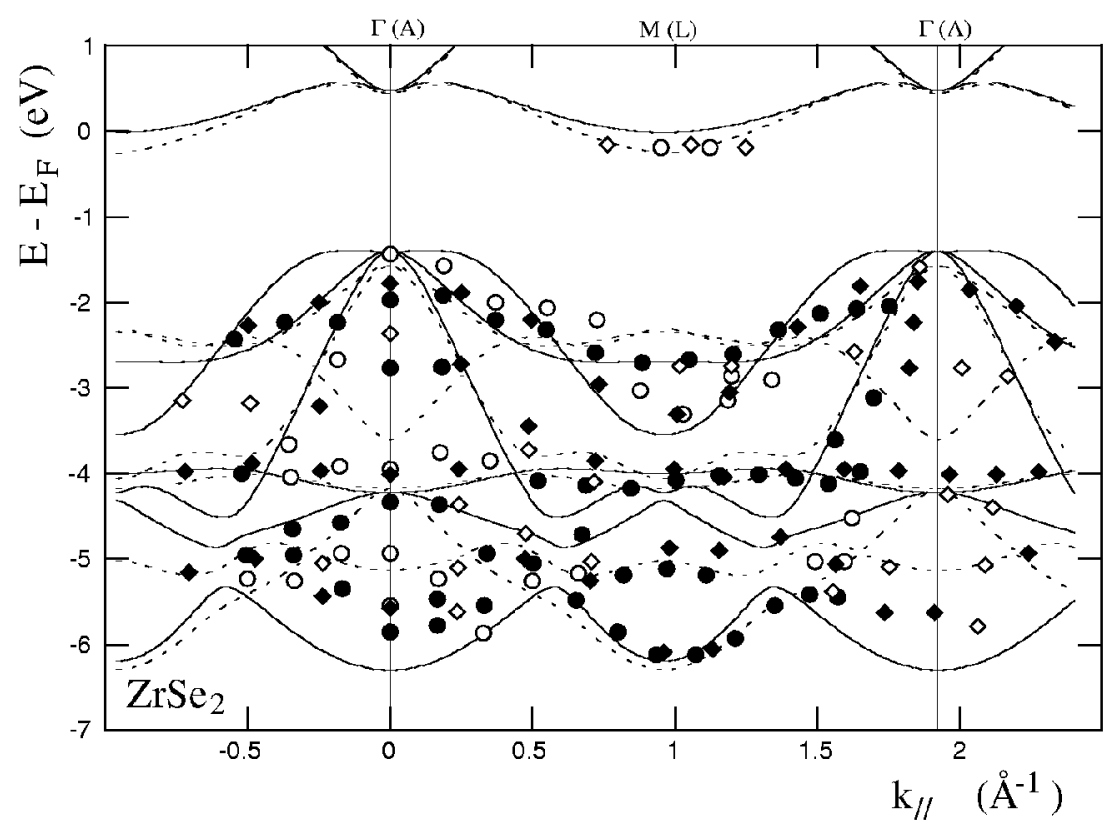

Fig. 5. Structure plot showing energy versus $k_{\|}$for the spectral features in Fig. 4 (circles) and from a similar series measured with $h \nu=38 \mathrm{eV}$ (squares). Filled symbols correspond to conspicious peaks and open ones to weaker structures. Comparisons are made with $\mathrm{ZrSe}_{2} \mathrm{LAPW}$ bands calculated along $\Gamma M$ (full lines) and $A L$ (dashed lines).

$\theta$ in the $\Gamma M$ azimuthal direction [8]. By plotting the initial state energy versus $k_{\|}$ for each spectral peak, one obtains a structure plot, as shown in Fig. 5, where the experimental points can be directly compared with the calculated band structure. The comparison is here done with two sets of bands, one (full lines) corresponding to the $\Gamma M$ line $\left(k_{\perp}=0\right)$ and the other set (dashed lines) corresponding to the $A L$ line $\left(k_{\perp}=\pi / c\right)$. The difference between the two sets is due to the perpendicular dispersion. For some bands the perpendicular dispersion is small, and here the comparison with the experimental points is very straightforward. For bands with a larger perpendicular dispersion one should be aware that also points falling between corresponding full and dashed lines are compatible with the calculations, as $k_{\perp}$ may assume any intermediate values. Self-consistent $a b$ initio band structure calculations by the linear augmented plane wave (LAPW) method are generally found to be in good agreement with experimental results from layered TMDCs, although the calculated band gap often is somewhat smaller than the one observed experimentally.

Although layered TMDCs are sometimes referred to as "two-dimensional" materials, this terminology is not strictly true, as these materials usually have some electronic bands with very significant perpendicular dispersion [9-11]. This 
dependence on $k_{\perp}$ can be studied by e.g. measuring spectra at normal emission $(\theta=0)$ for different photon energies. As the photon energy is changed, the value of $k_{\perp}$ for a transition from a certain initial band to a certain final band also changes, and the perpendicular dispersion of the initial band will then result in a changed position of the spectral peak on the initial energy scale. However, to map the perpendicular dispersion of the initial bands accurately, one need in principle to know the dispersion of the final bands. A common approximation in this context is to assume the final band to be a free-electron parabola, shifted in energy by an adjustable "inner potential". With a suitable choice of inner potential it is often possible to obtain a reasonably accurate mapping of the perpendicular dispersion for metals. In layered materials, however, the strong anisotropy results in large deviations from the simple parabolic shape for the final bands, and the approximation may therefore produce directly misleading results. Also in ordinary semiconductor materials the final bands may deviate significantly from the free-electron parabolic shape, since the crystal potential in these is generally stronger than in metals. One way out of these difficulties is the recently developed method of very-low-energy electron diffraction (VLEED) [12], by which it is possible to obtain accurate final bands for analysis of ARPES data.

The VLEED method is based on the measurement of the elastic electron reflectivity $R(E)$ at kinetic energies in the range of $0-40 \mathrm{eV}$. This can be done using an ordinary LEED apparatus arranged as shown in Fig. 6 [13]. The exit electrode of the electron gun is kept at a fixed positive voltage relative to the cathode, and the kinetic energy of the electron beam is adjusted by biasing of the cathode with the sample at ground potential. With this arrangement a constant beam current $I_{0}$ can be maintained down to the lowest energies. In practice it is more convenient to measure the absorbed current $I_{T}$ as a function of energy. Since the transmission

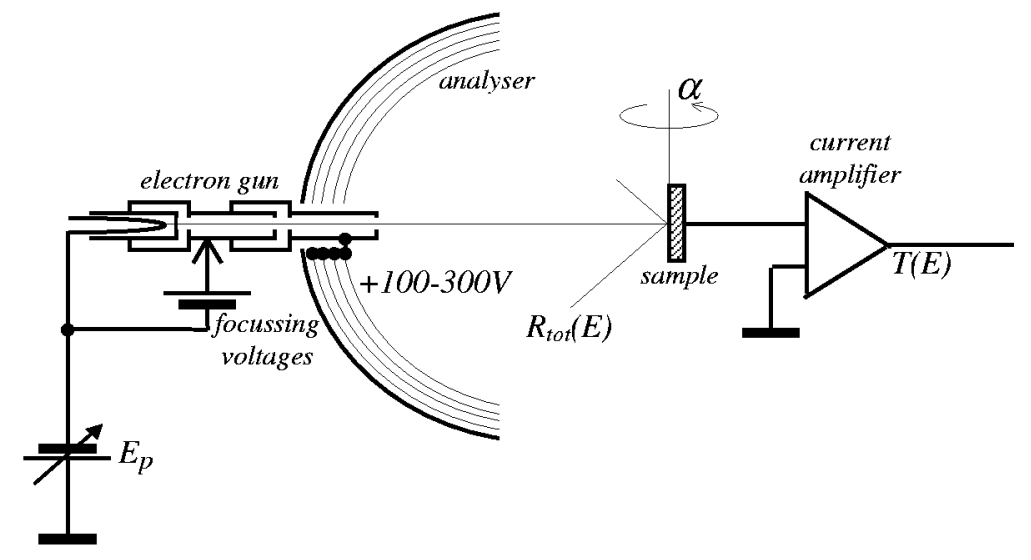

Fig. 6. Configuration of standard LEED equipment for VLEED measurements (after Strocov [13]). 


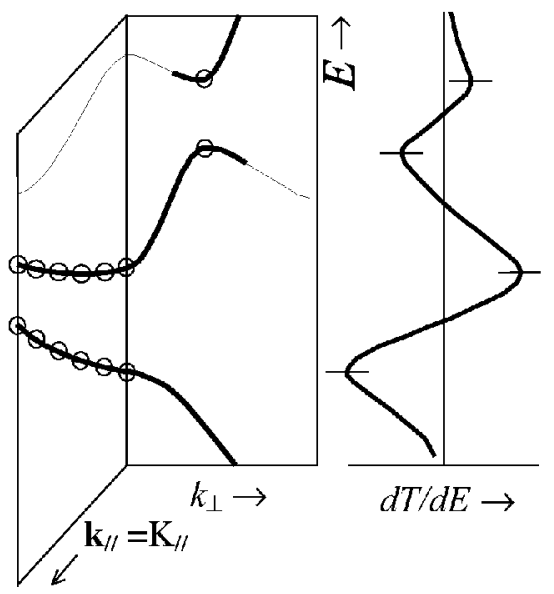

Fig. 7. Principles of VLEED band determination: critical points in the band structure correspond to maxima and minima in the $\mathrm{d} T / \mathrm{d} E$ curve (after Strocov [13]).

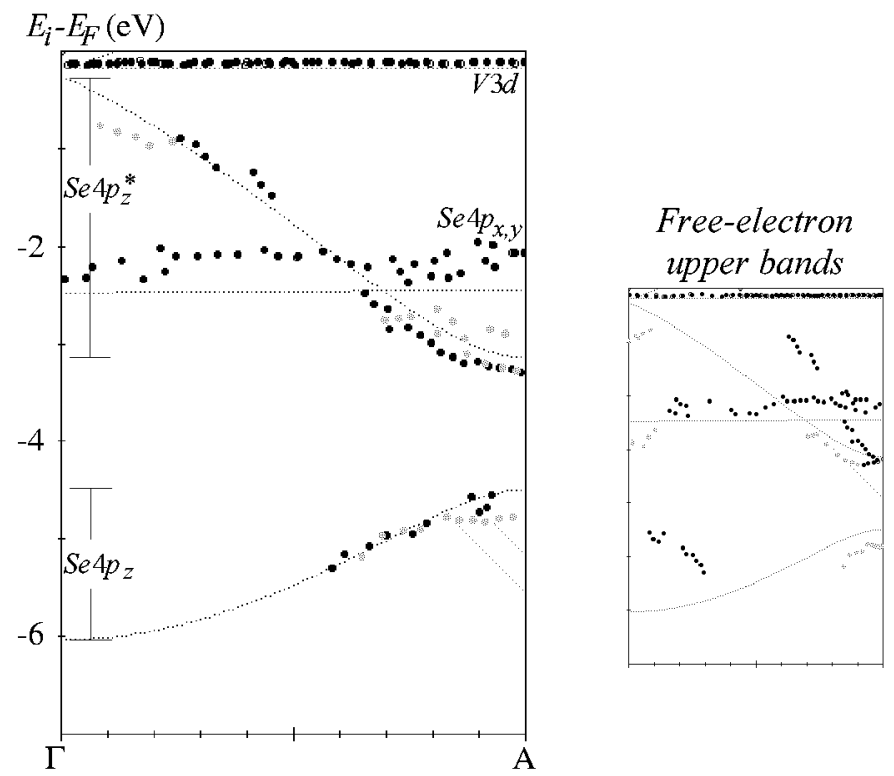

Fig. 8. ARPES mapping of the $\mathrm{VSe}_{2}$ valence bands using final bands determined by VLEED. The experimental dots are imposed on bands calculated by the LAPW method. Black dots are determined more accurately than grey dots. The smaller panel to the right shows the largely erroneous results obtained with free-electron-like final bands (based on data from Strocov et al. [14]). 
$T(E)=I_{T} / I_{0}=1-R(E)$, this function contains the same information as $R(E)$. VLEED is connected with the band structure through the fact that the transmission of incident electrons with a certain energy depends on if there are suitable Bloch states available in the solid at this energy. More precisely, one finds that the maxima and minima of the derivative $\mathrm{d} T / \mathrm{d} E$ correspond to critical points in the band structure, as illustrated in Fig. 7. The band structure in the perpendicular direction can therefore be determined, e.g. by fitting a parametrized band structure to the critical points, and the bands in the parallel direction can be mapped directly by varying the incidence angle (and hence $k_{\|}$).

Bands detectable by VLEED have to fulfill two conditions: they must be above the vacuum level, and the Bloch waves in the solid should have a strong coupling to the plane waves in vacuum. These are exactly the same conditions which are required for the final states in photoenmission, which makes the combination of VLEED and ARPES particularly powerful for studies of perpendicular dispersion. As an example, Fig. 8 shows the bands along the $\Gamma A$ line in $\mathrm{VSe}_{2}$ mapped by the use of VLEED and free-electron-like final bands, respectively [14].

\section{Intercalation of layered TMDCs}

A particularly interesting property of TMDCs is that foreign atoms or molecules can be inserted between the host layers [15]. This is known as intercalation, and can be employed to modify the physical properties of the material in a controllable way $[4,16]$. It is for instance possible to achieve semiconductor-to-metal transitions (or the opposite) by intercalation.

Many of the changes occurring upon intercalation are due to charge transfer from the introduced species to the host lattice, and this is often described in terms of the rigid band model (RBM), in which it is assumed that the band structure of the host material is unchanged, except that the band filling is altered by the charge transfer.

Another important effect of intercalation is the increased spacing between the layers, which is necessary to accommodate the intercalated species. This will affect the degree of anisotropy, and may produce changes incompatible with the RBM. In addition there may also occur charge-transfer induced structural changes (of bond lengths, coordinations and superstructures) within the layers, or changes in layer stacking because of modified interlayer interactions.

The three most important categories of TMDC intercalants are:

(i) alkali metals and other simple metals, including $\mathrm{Cu}$ and $\mathrm{Ag}$ [17];

(ii) $3 d$ transition metals [18];

(iii) ammonia, hydrazine, and related organic molecules [2].

This review deals only with alkali metal intercalation, although many effects are similar for the different types of intercalants. Intercalation of TMDCs with alkali metals can be achieved by a number of methods, e.g. immersion in 
alkali-ammonia solutions, electrolysis of a solution of an appropriate metal salt (with the TMDC sample acting as cathode), or immersion in buthyl-lithium $\left(n-\mathrm{C}_{4} \mathrm{H}_{9} \mathrm{Li}\right.$ ). However, the most convenient method in combination with electron spectroscopies is in situ intercalation through alkali metal deposition in ultra-high vacuum (UHV) onto clean TMDC surfaces. In most cases the deposited alkali metals intercalates spontaneously without significant degradation of the surface, and by doing e.g. ARPES measurements on the same sample before and after the intercalation, one may study the effect of intercalation on the electronic structure in detail.

Figure 9 shows spectra measured from the same $\mathrm{ZrSe}_{2}$ sample as in Fig. 4, but after in situ intercalation with Cs, and Fig. 10 shows the corresponding structure

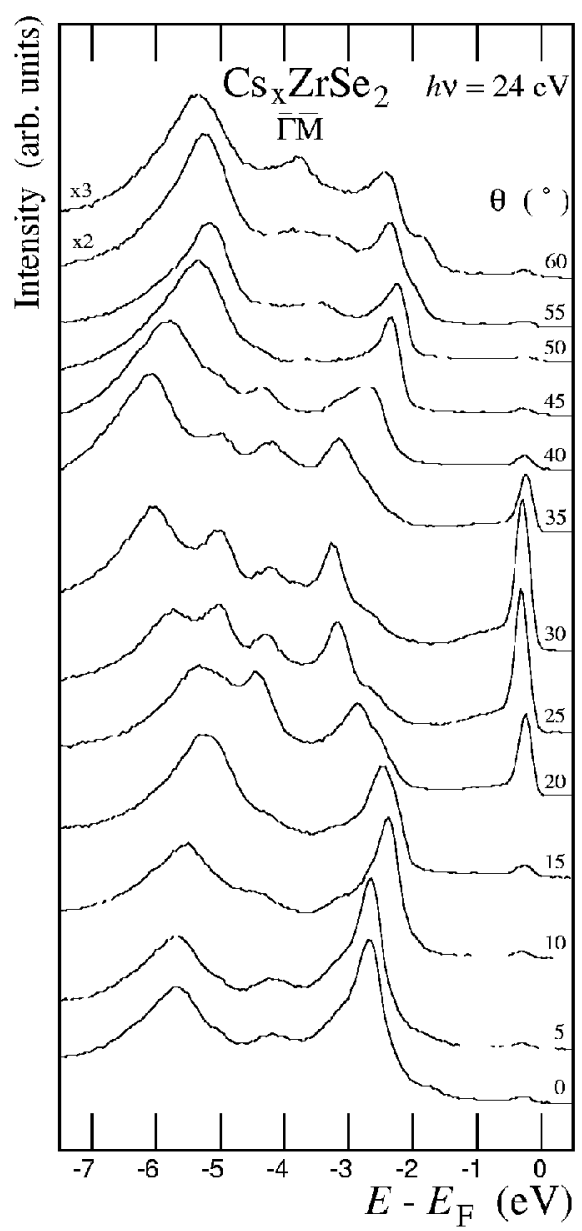

Fig. 9. Angular series of photoelectron spectra measured from $\mathrm{Cs}_{x} \mathrm{ZrSe}_{2}$ with $h \nu=$ $24 \mathrm{eV}$. 


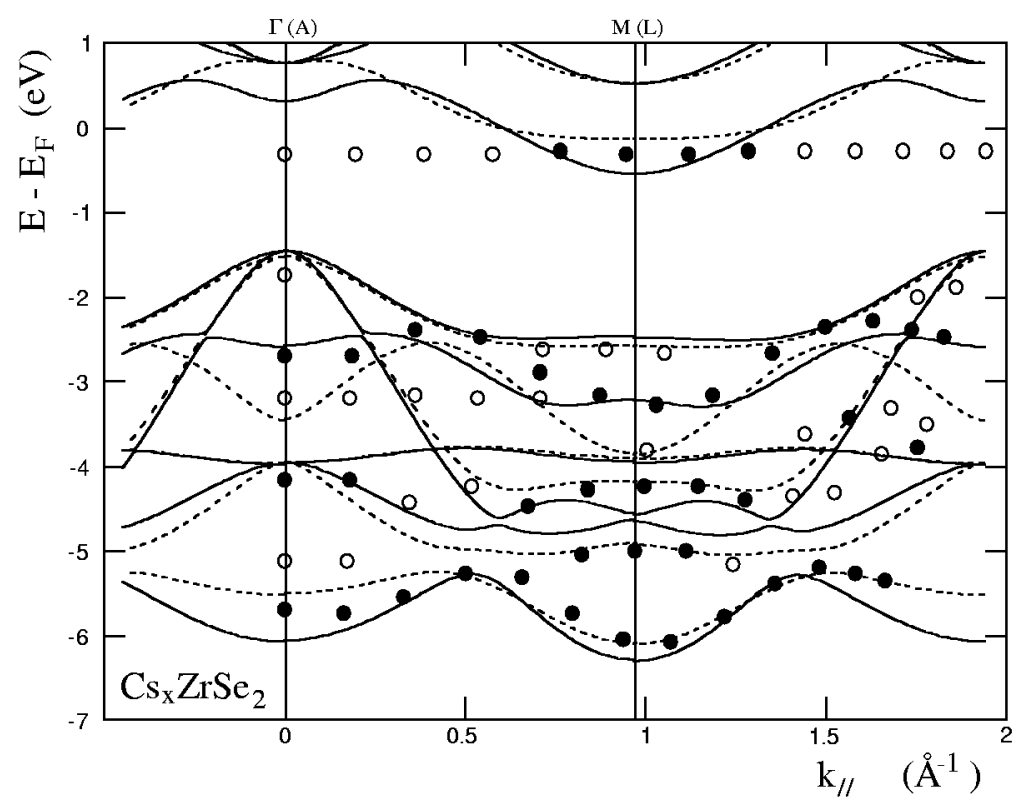

Fig. 10. Structure plot showing energy versus $k_{\|}$for the spectral features in Fig. 9. Filled circles correspond to conspicious peaks and open ones to weaker structures. Comparisons are made with $\mathrm{CsZrSe}_{2} \mathrm{LAPW}$ bands calculated along $\Gamma M$ (full lines) and $A L$ (dashed lines).

plot compared with LAPW calculations [8]. A notable intercalation induced feature in Fig. 9 is the large peak occurring just below the Fermi level for polar angles around $30^{\circ}$. This peak reveals that the lowest conduction band (of $\mathrm{Zr} 4 d$ origin) has become partially occupied by electrons transferred from the Cs to the host layers, transforming the sample from a semiconductor to a metal. Again good agreement is found with the calculated band structure (after manual adjustment of the band gap), but it is also clear that the changes to the band structure are not just a matter of band filling, since there are significant differences (especially regarding the perpendicular dispersion) which goes well beyond the RBM. Similar results have been obtained from several other alkali/TMDC systems such as $\mathrm{Na} / \mathrm{TiS}_{2}$, $\mathrm{Cs} / \mathrm{TiS}_{2}$ [19], $\mathrm{Na} / \mathrm{VSe}_{2}, \mathrm{~K} / \mathrm{VSe}_{2}, \mathrm{Cs} / \mathrm{VSe}_{2}$ [20], Na/2H-TaS 2 and $\mathrm{Cs} / 2 \mathrm{H}-\mathrm{TaS}_{2}$ [21]. In all cases the main effects of alkali metal intercalation were an increase in band filling, reduced perpendicular dispersion and significant non-RBM changes in the finer details. In order to fully understand these intercalation induced changes, it is very important to perform full $a b$ initio band structure calculations.

In addition to the observations quoted above, the ARPES results also showed some deviations from the calculated band structures which possibly could be attributed to defects. There are several ways in which intercalation may create defects. As the lattice parameters are altered by the intercalation, in particular the 


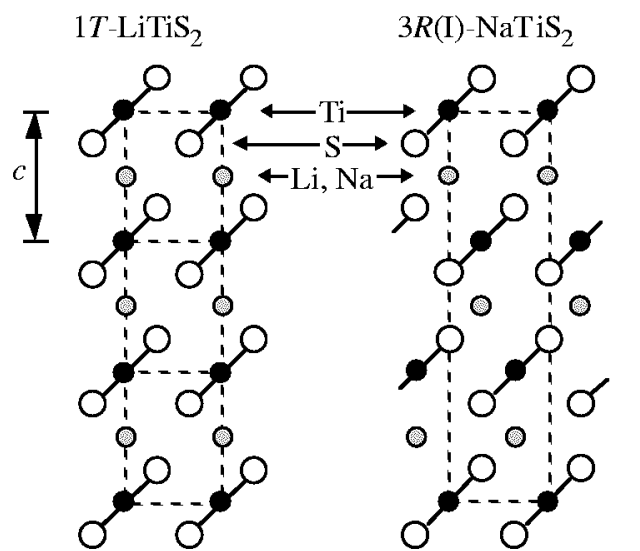

(a) (b)

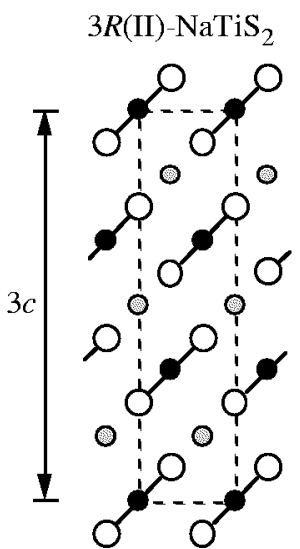

(c)

Fig. 11. Different layer stackings in $\mathrm{TiS}_{2}$ intercalated with alkali metals.

layer spacing $c$, but also the in-plane parameter $a$, the layers will be severely strained during intercalation and cracks may form if the stress is high enough. Such cracks have been verified in studies of the $\mathrm{Cs} / \mathrm{TiS}_{2}$ system by transmission electron microscopy (TEM) [22]. Another possible effect of intercalation is that it may change the stacking of the layers [3]. Whether such changes are favourable or not depends on the size of the alkali metal ions. Figure 11 shows how the $1 T$ structure of $\mathrm{TiS}_{2}$ is retained upon intercalation with $\mathrm{Li}$, while intercalation with $\mathrm{Na}$ may result in two different types of $3 R$ stacking. During in situ intercalation of large crystals, such structure transformations may be incomplete due to defects and inhomogeneities which partially pin the layers together. This may then result in stacking disorder which affect the perpendicular dispersion in ARPES measurements. Evidence for such incomplete stacking changes and disorder has been found by both TEM [22] and incoherent medium-energy electron diffraction [23]. These findings implies that disorder, inhomogeneities, and other defects are of significant importance in intercalation processes.

\section{Metallic overlayers on layered semiconductors}

Layered TMDCs are easily cleaved to produce high-quality (0001) surfaces, which have no dangling bonds and therefore are chemically inert with no surface states. In principle, surfaces of semiconducting TMDCs should be ideal for studies of Schottky barriers. A practical problem is that the weak interaction between the surface and adsorbed metals favours the growth of three-dimensional metal clusters. In order to obtain homogeneous metal overlayers it may be necessary to keep the sample at low temperature. Another problem is of course the ability of many metals to intercalate, which in this context leads to doping of the semiconductor 


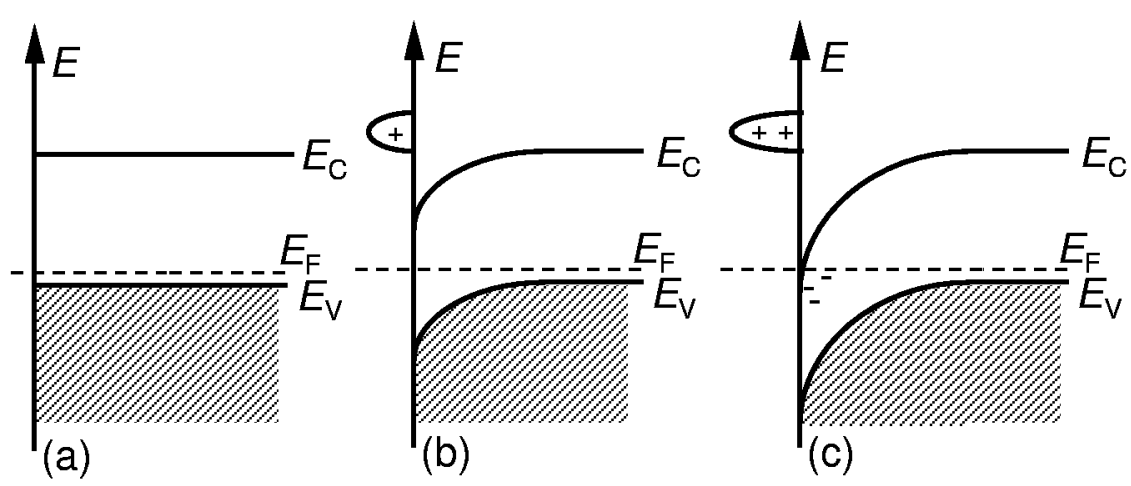

Fig. 12. Schematical illustration of band bending in $p$-WSe $\mathrm{W}_{2}$ induced by $\mathrm{Rb}$ deposition: (a) initial flat band conditions; (b) moderate band bending with a depletion layer; (c) saturated band bending with an inversion layer.

and formation of interfaces which are not abrupt. So deviations from ideal Schottky behaviour for the $\mathrm{Cu} / \mathrm{WSe}_{2}$ and $\mathrm{Ag} / \mathrm{WSe}_{2}$ interfaces have been attributed to intercalation [24]. One system where intercalation does not seem to occur is $\mathrm{Rb} / \mathrm{WSe}_{2}$. One group at the University of Kiel has studied in detail the band bending and surface photovoltage effect at submonolayer Rb coverage [25]. They measured how the valence band spectra of $p$-type $\mathrm{WSe}_{2}$ shifted as the Rb coverage was gradually increased. The nature of these shifts are illustrated in Fig. 12. Figure 12a shows the initial flat band conditions typical of semiconductors surfaces without surface states. In (b) a small amount of $\mathrm{Rb}$ has been deposited, and as the $\mathrm{Rb} 5 s$ states are above the Fermi level, its valence electrons are transferred to the substrate. The result is band bending in the depletion layer. The band bending increases as more $\mathrm{Rb}$ is added, until the conduction band minimum reaches the Fermi level, as illustrated in (c). Now one obtains an inversion layer with electrons populating the conduction band, and a further $\mathrm{Rb}$ deposition just increases the conduction band population, without significantly increasing the band bending. This picture is confirmed by the spectra in Fig. 13, which shows the band bending to saturate at $1.1 \mathrm{eV}$ for $\mathrm{Rb}$ coverages exceeding $\sim 10^{12}$ atoms $/ \mathrm{cm}^{3}$. Figure 14 shows an additional effect due to the surface photovoltage. The upper spectrum in (a) was measured from the clean $\mathrm{WSe}_{2}$ surface, and the middle one after the $\mathrm{Rb}$ deposition (nominally $0.5 \times 10^{12}$ atoms $/ \mathrm{cm}^{2}$ ), which gave rise to a band bending of $519 \mathrm{meV}$. Then the sample was illuminated by white light $\left(332 \mathrm{~mW} / \mathrm{cm}^{2}\right)$, which caused the spectrum to shift back by $169 \mathrm{meV}$ (bottom curve). The reason for this shift is that the illumination generates electron-hole-pairs, and that the additional electrons in the conduction band reduce the band bending required to compensate the positive $\mathrm{Rb}$ charge. Such surface photovoltage shifts can be distinguished from the band bending shifts by their dependence on the illumination level, which was also studied by the Kiel group. In Fig. 14b the dots show the total 


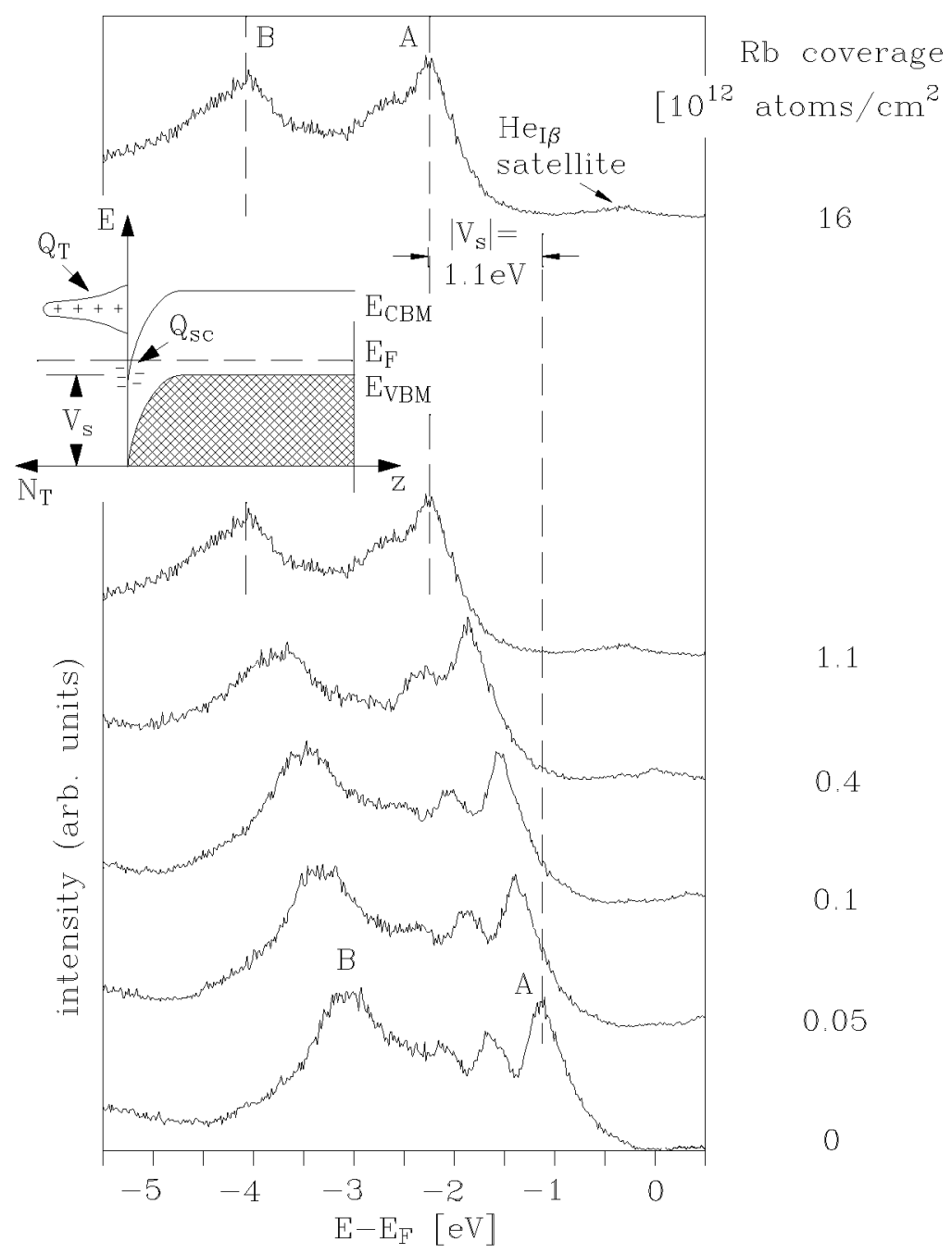

Fig. 13. Rb induced band bending in $p-\mathrm{WSe}_{2}$ as observed by photoelectron spectroscopy. Reproduced from [25] with permission from Elsevier Science (Copyright 1998).

shift at different illumination levels, which correspond to different electron-hole generation rates $G_{0}$. The solid line is the result of a numerical calculation based on semiclassical semiconductor theory. The excellent agreement confirms the ideal nature of the studied surface.

The same group at University of Kiel has also discovered that $\mathrm{Rb}$, when deposited on several TMDCs (among them $\mathrm{WSe}_{2}$ ) under certain conditions, forms nanowire networks with a typical mesh size of the order of microns $[26,27]$. The implications of this for future nanostructure engineering of surfaces are yet to be settled, but the prospects are encouraging. 

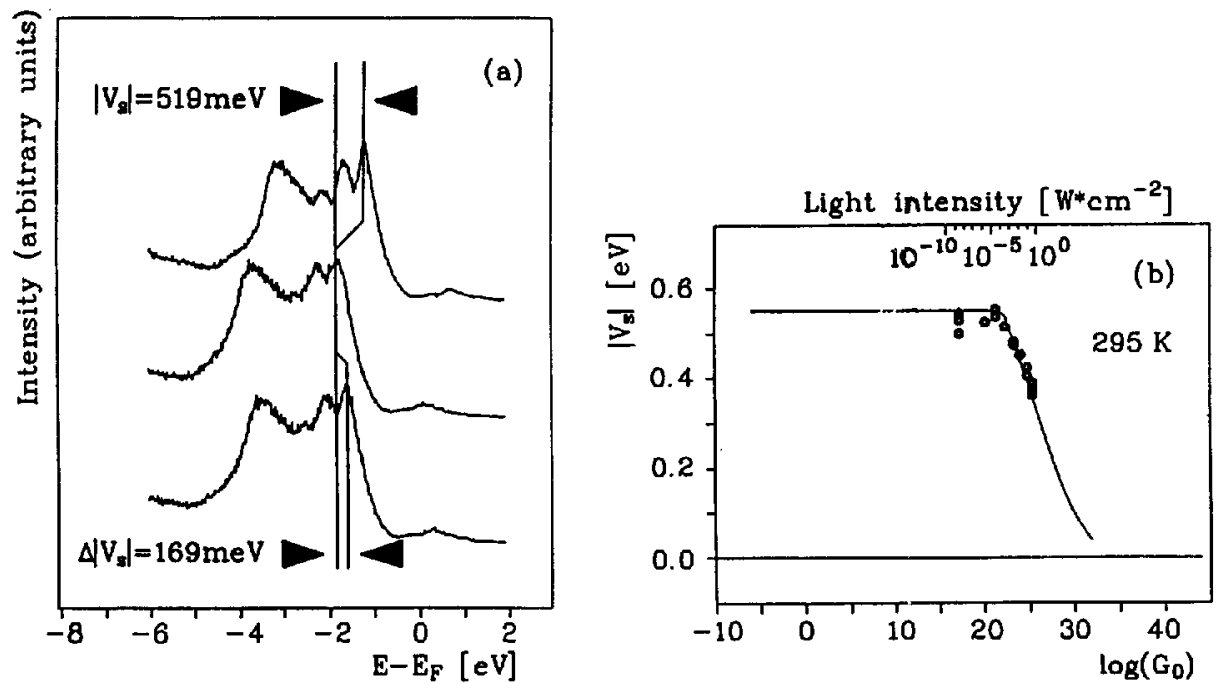

Fig. 14. (a) Reduced band bending due to the surface photovoltage effect. (b) The dots show the observed band bending as a function of illumination intensity. The solid line shows the band bending as calculated from semiclassical semiconductor theory. Reproduced from [25] with permission from Elsevier Science (Copyright 1998).

\section{Interface formation by van der Waals epitaxy}

The term "van der Waals epitaxy" was introduced by Koma et al. [28] to describe the growth of a layered compound when deposited on the cleavage surface of another layered compound. This topic has recently been thoroughly reviewed by Jaegermann et al. [29]. Like in ordinary epitaxial growth it is possible to grow well ordered and oriented overlayers of these materials, but the growth does not require lattice matching. Due to the weak interactions and absence of directed bonds between the layers, the overlayer may grow with a radically different periodicity as illustrated in Fig. 15. In studies of such deposited overlayers by scanning tunneling microscopy (STM), the difference in periodicity may be seen as Moiré-type height undulations (Fig. 16) [30].

This type of epitaxy opens new possibilities for studies of e.g. band line-up at semiconductor interfaces. Just like for surfaces, the resulting interfaces are almost free from dangling bonds and interface states, making them ideal for studies of fundamental interface theory. The freedom from lattice matching constraints allows for a huge number of material combinations.

It is also possible to grow layered materials epitaxially on top of three-dimensional materials (e.g. GaAs or other conventional semiconductors), or vice versa. This kind of growth, which is called "quasi-van der Waals epitaxy" is illustrated in Fig. 17. In principle, it might be possible to interface lattice mismatched three-dimensional semiconductors by growing a buffer layer between them by quasi-van der Waals epitaxy. 


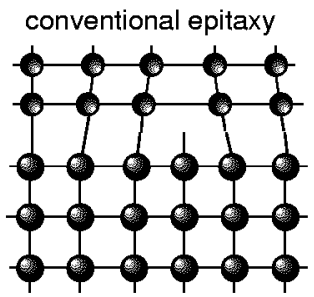

van der Waals-epitaxy

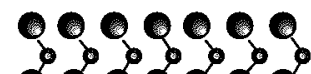

ค.

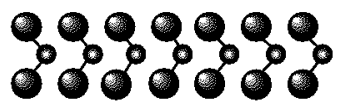

Fig. 15. Lattice mismatch in conventional and van der Waals epitaxy, respectively. Reproduced from [29] with permission from Kluwer Academic Publishers (Copyright 2000).

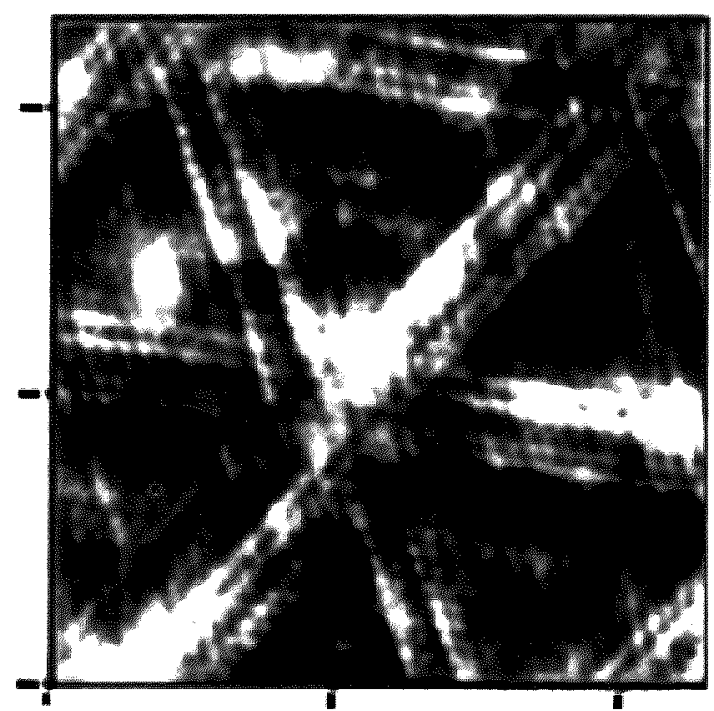

Fig. 16. Moiré structure of $\mathrm{MoSe}_{2}$ on $\mathrm{MoS}_{2}$ as seen by STM. Reproduced from [30] with the authors' permission.
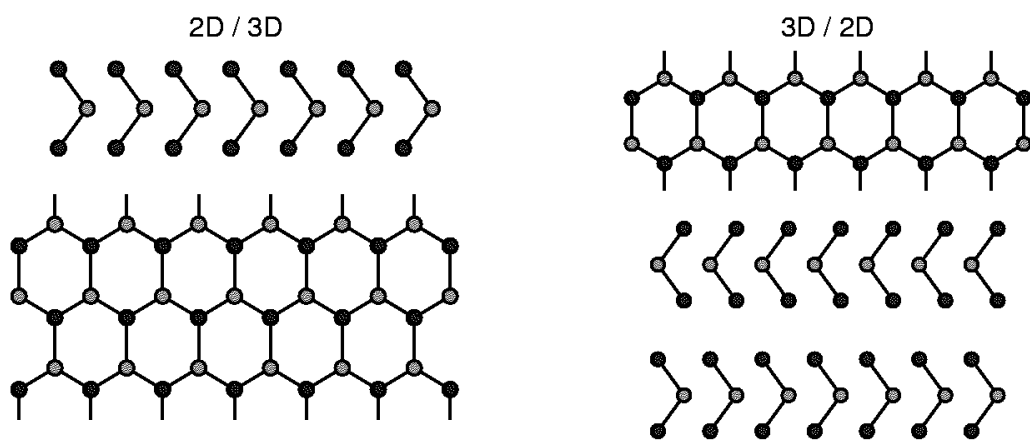

Fig. 17. Combinations of two- and three-dimensional materials by "quasi-van der Waals epitaxy". Reproduced from [29] with kind permission from Kluwer Academic Publishers (Copyright 2000). 
For practical applications, van der Waals and quasi-van der Waals epitaxy may thus allow for design of novel thin film semiconductor devices. One problem here is the complex nature of the growth morphology, which may be radically different for different material combinations. To master this will certainly be a key element for successful development of these new techniques.

\section{Applications in photovoltaics}

Several layered semiconductors are of interest for solar cell applications [31]. $\mathrm{WSe}_{2}$ is one of the leading candidates in this context, with an achieved conversion efficiency of $8 \%$. In principle it should be possible to increase this efficiency considerably, as much higher efficiencies have been obtained in electrochemical cells based on the same material. The inertness of the layers is an important advantage of TMDCs, and a further advantage of $\mathrm{WSe}_{2}$ is that the fundamental band gap is between non-bonding $d$ states (in analogy with $\mathrm{MoS}_{2}$, Fig. 2b), which means that the photoexcitation will not weaken the bonding. $\mathrm{WSe}_{2}$ and isoelectronic materials are therefore resistive against photocorrosion. The problems of using layered semiconductors in solar cells are largely practical, e.g. how to prepare thin films of a sufficient quality in sufficiently large dimensions. The technique of van der Waals epitaxy mentioned above, may be useful here. Still, the layered semiconductors are of great interest in solar cell model studies, through their ideal nature of their surfaces.

\section{References}

[1] J.A. Wilson, A.D. Yoffe, Adv. Phys. 18, 193 (1969).

[2] G.V. Subba Rao, M.W. Shafer, in: Intercalated Layered Materials, Ed. F.A. Lévy, D. Reidel, Dordrecht 1979, p. 99.

[3] J. Rouxel, in: Intercalated Layered Materials, Ed. F.A. Lévy, D. Reidel, Dordrecht 1979, p. 201.

[4] W.Y. Liang, in: Intercalation in Layered Materials, Ed. M.S. Dresselhaus, Plenum, New York 1986, p. 31.

[5] Structural Phase Transitions in Layered Transition Metal Compounds, Eds. K. Motizuki, D. Reidel, Dordrecht 1986.

[6] Angle-Resolved Photoemission, Ed. S.D. Kevan, Elsevier, Amsterdam 1992.

[7] S. Hüfner, Photoelectron Spectroscopy, 2nd ed., Springer, Berlin 1996.

[8] H.E. Brauer, H.I. Starnberg, L.J. Holleboom, H.P. Hughes, J. Phys., Condens. Matter 7, 7741 (1995).

[9] O. Anderson, R. Manzke, M. Skibowski, Phys. Rev. Lett. 55, 2188 (1985).

[10] H.I. Starnberg, H.E. Brauer, L.J. Holleboom, H.P. Hughes, Phys. Rev. Lett. 70, 3111 (1993).

[11] V.N. Strocov, H.I. Starnberg, P.O. Nilsson, H.E. Brauer, L.J. Holleboom, Phys. Rev. Lett. 79, 467 (1997). 
[12] V.N. Strocov, H.I. Starnberg, P.O. Nilsson, Phys. Rev. B 56, 1717 (1997).

[13] V.N. Strocov, in: Electron Spectroscopies Applied to Low-Dimensional Materials, Eds. H.P. Hughes, H.I. Starnberg, Kluwer, Dordrecht 2000, p. 161.

[14] V.N.Strocov, H.I. Starnberg, P.O. Nilsson, H.E. Braner, L.J. Holleboom, J. Phys., Condens. Matter 10, 5749 (1998).

[15] Intercalated Layered Materials, Ed. F.A. Lévy, D. Reidel, Dordrecht 1979.

[16] R.H. Friend, A.D. Yoffe, Adv. Phys. 36, 1 (1987).

[17] H.I. Starnberg, H.E. Brauer, H.P. Hughes, in: Electron Spectroscopies Applied to Low-Dimensional Materials, Eds. H.P. Hughes, H.I. Starnberg, Kluwer, Dordrecht 2000 , p. 41.

[18] M. Inoue, H.P. Hughes, A.D. Yoffe, Adv. Phys. 38, 565 (1989).

[19] H.E. Brauer, H.I. Starnberg, L.J. Holleboom, H.P. Hughes, V.N. Strocov, J. Phys., Condens. Matter 11, 8957 (1999).

[20] H.E. Brauer, H.I. Starnberg, L.J. Holleboom, V.N. Strocov, H.P. Hughes, Phys. Rev. B 58, 10031 (1998).

[21] H.E. Brauer, H.I. Starnberg, L.J. Holleboom, H.P. Hughes, V.N. Strocov, submitted to J. Phys., Condens. Matter.

[22] M. Remškar, A. Popović, H.I. Starnberg, Surf. Sci. 430, 199 (1999).

[23] I.I. Pronin, M.V. Gomoyunova, N.S. Faradzhev, D.A. Valdaitsev, H.I. Starnberg, Surf. Sci. 461, 137 (2000).

[24] W. Jaegermann, C. Pettenkofer, B.A. Parkinson, Phys. Rev. B 42, 7487 (1990).

[25] M. Boehme, R. Adelung, M. Traving, L. Kipp, M. Skibowski, Appl. Surf. Sci. 123/124, 91 (1998).

[26] R. Adelung, L. Kipp, J. Brandt, L. Tarcak, M. Traving, C. Kreis, M. Skibowski, Appl. Phys. Lett. 74, 3053 (1999).

[27] R. Adelung, J. Brandt, K. Rossnagel, O. Seifarth, M. Skibowski, C. Ramirez, T. Strasser, W. Schattke, Phys. Rev. Lett. 86, 1303 (2001).

[28] A. Koma, K. Sunouchi, T. Miyajima, J. Vac. Sci. Technol. B 3, 724 (1985).

[29] W. Jaegermann, A. Klein, C. Pettenkofer, in: Electron Spectroscopies Applied to Low-Dimensional Materials, Eds. H.P. Hughes, H.I. Starnberg, Kluwer, Dordrecht 2000 , p. 317.

[30] B.A. Parkinson, F.S. Ohuchi, K. Ueno, A. Koma, Appl. Phys. Lett. 58, 472 (1991).

[31] Photoelectrochemistry and Photovoltaics of Layered Semiconductors, Ed. A. Aruchamy, Kluwer, Dordrecht 1992. 